\title{
Therapeutic Cocktail Approach for Treatment of Hyperhomocysteinemia in Alzheimer's Disease
}

\author{
Michael Leon', Darrell Sawmiller', R. Douglas Shytle², and Jun Tan'
}

\begin{abstract}
In the United States, Alzheimer's disease (AD) is the most common cause of dementia, accompanied by substantial economic and emotional costs. During 2015, more than 15 million family members who provided care to AD patients had an estimated total cost of 22I billion dollars. Recent studies have shown that elevated total plasma levels of homocysteine (tHcy), a condition known as hyperhomocysteinemia ( $\mathrm{HHcy}$ ), is a risk factor for AD. HHcy is associated with cognitive decline, brain atrophy, and dementia; enhances the vulnerability of neurons to oxidative injury; and damages the blood-brain barrier. Many therapeutic supplements containing vitamin $\mathrm{BI} 2$ and folate have been studied to help decrease tHcy to a certain degree. However, a therapeutic cocktail approach with 5-methyltetrahydrofolate, methyl BI2, betaine, and $\mathrm{N}$-acetylcysteine (NAC) have not been studied. This novel approach may help target multiple pathways simultaneously to decrease tHcy and its toxicity substantially.
\end{abstract}

\section{Keywords}

Alzheimer's disease, betaine, vitamin $B \mid 2$, folate, $N$-acetylcysteine (NAC), reactive oxygen species (ROS), glutathione (GSH), hyperhomocysteinemia (HHcy), total homocysteine (tHcy)

\section{Introduction}

Alzheimer's disease (AD) is the most common form of dementia that consists of decline in memory, language, and problemsolving skills. AD accounts for more than $50 \%$ of all clinically diagnosed dementia. ${ }^{1}$ People in the final stages of $\mathrm{AD}$ are bed bound and require constant care, ultimately leading to death. ${ }^{2}$ By 2050, a new case of AD is expected to occur every $33 \mathrm{~s}$, causing nearly 1 million new AD cases per year. The pathophysiology of AD involves neurons being damaged or destroyed. ${ }^{2}$ After many years of research, studies have shown that elevated homocysteine (Hcy) is a major risk factor. Hcy promotes excitotoxicity and gives rise to neuronal DNA damage and apoptosis, thereby affecting short-term memory and learning. It has become clear that elevated levels of Hcy elicit neuronal death, including hippocampal and cortical neurons. ${ }^{1,3}$ Cross-sectional magnetic resonance imaging (MRI) studies describe a similar correlation between high Hcy levels and cerebral atrophy. The increased level of tHcy predicts the rate of shrinkage of the medial temporal lobe in patients with AD. ${ }^{4}$

\section{Hyperhomocysteinemia (HHcy)/Oxidative Stress}

HHcy is an elevation in blood levels of Hcy, a sulfurcontaining nonproteinogenic amino acid. ${ }^{5}$ Hcy is derived from the hydrolysis of $S$-adenosyl Hcy during methionine (Met) metabolism. ${ }^{6}$ Although Hcy is a normal metabolite, Hcy excess is a risk factor for cognitive impairment and dementia. ${ }^{7}$ HHcy has been associated with various adverse health conditions including thrombosis, neuropsychiatric illness, fractures, and cardiovascular disease. ${ }^{8}$ While the serum level of Hcy in healthy adults ranges from 5 to $10 \mu \mathrm{M}$, mild Hcy ranges from 15 to $20 \mu \mathrm{M}$ but can rise up to $500 \mu \mathrm{M}$, and major symptoms have been shown to include neurodegenerative pathologies such as dementia, Parkinson's disease (PD), and AD. ${ }^{9}$ Reports show that older adults with HHcy have more regional brain atrophy and reduced volumes of medial

\footnotetext{
' Department of Psychiatry and Behavioral Neurosciences, Rashid Laboratory for Developmental Neurobiology, Silver Child Development Center, Morsani College of Medicine, University of South Florida, Tampa, FL, USA

2 Department of Neurosurgery and Brain Repair, Center for Excellence in Aging and Brain Repair, Morsani College of Medicine, University of South Florida, Tampa, FL, USA

Corresponding Author:

Michael Leon, Department of Psychiatry and Behavioral Neurosciences, Rashid Laboratory for Developmental Neurobiology, Silver Child Development Center, Morsani College of Medicine, University of South Florida, I290I Bruce B Downs Blvd., MDC 19 Tampa, FL 336I2, USA. Email: mleon@health.usf.edu
} 
orbitofrontal cortices. In addition, they have thinner cortical gray matter on MRI. ${ }^{10}$

Hcy is an excitatory amino acid that enhances the vulnerability of neurons to oxidative injury. Oxidative stress has been implicated in the disease progression of AD and PD. Oxidative stress is the cause of unregulated production of reactive oxygen species (ROS). ROS can initiate neuronal cell death, causing neurodegeneration. For example, highly reactive hydroxyl radical is considered one of the ROS that can subsequently lead to lipid peroxidation, protein oxidation, and protein aggregation. ${ }^{1-16}$ The brain is especially susceptible to oxidative stress because of its relatively low antioxidant enzymes. ${ }^{17}$ In addition, elevated total Hcy has an increased risk for other disease, such as vascular diseases and dementia. $^{18}$

Furthermore, Hcy acts as a glutamate agonist on the $N$-methyl-D-aspartate (NMDA) receptor. Overstimulation of this receptor results in an increased level of cytoplasmic calcium and higher production of free radicals and leads to apoptosis. ${ }^{19-21}$ Intracerebroventricular injections of Hcy in rats have induced lipid peroxidation, severe oxidative stress, neuroinflammation, and cognitive impairment. ${ }^{22}$ HHcy also affects the microvasculature and the blood-brain barrier (BBB), which is more stringently controlled in the cortex. The microcirculation of the brain is more susceptible to disruption by HHcy, which causes increased expression of matrix metalloproteinases (MMPs). In the heterozygous cystathionine- $\beta$-synthase (CBS) knockout mouse model, the resulting HHcy increased brain permeability in relation to increased MMP activity, extracellular matrix degradation, vascular inflammation, and endothelial dysfunction. ${ }^{22}$ Several proinflammatory agents, such as endotoxins, cytokines, and other oxidative stress factors, upregulate MMP activity in astrocytes in vitro. ${ }^{22}$ The accumulation of toxic free radicals plays a key role in $\mathrm{BBB}$ disruption via the increased activity of MMPs.

\section{N-Acetylcysteine (NAC)}

Glutathione (GSH) is a major endogenous antioxidant. Several studies have implicated GSH redox imbalance in $\mathrm{AD}$ and PD. Both animal models and humans with these disorders have demonstrated altered levels of GSH and oxidized GSH (GSSG), a decreased ratio of GSH/GSSG, and/or impaired expressions or activities of GSH-related enzymes in the brain. ${ }^{12,13,23}$

GSH deficiency is associated with many pathological conditions. However, administering NAC, a cysteine prodrug, helps increase intracellular GSH levels. NAC supplies the cysteine necessary for GSH synthesis. ${ }^{23-25}$ In particular, NAC increases the GSH levels in the brain and alleviates oxidative stress-associated damage. ${ }^{26} \mathrm{GSH}$ has been implicated in the protection of brain cells against ROS. ${ }^{27}$ Indeed, NAC is a remarkably active agent that has provided people with relief in a variety of clinical settings. ${ }^{28}$ For example, NAC reduces the duration of attacks of chronic obstructive pulmonary disease; helps treat human immunodeficiency virus; improves neuropsychiatric disorders; and offers antioxidant, anti-inflammatory, mucolytic, and hepatoprotective benefits. $^{29-31} \mathrm{NAC}$ itself is an amino-acid L-cysteine derivative; evidence supports that administering NAC helps reduce oxidative damage in $\mathrm{AD} .^{32}$ Oral NAC administration increases cerebral spinal fluid (CSF) NAC concentrations, with the highest total NAC concentration of $10.1 \pm 0.8 \mu \mathrm{M}^{33}$

GSH is depleted in the early stages of PD, and deficiency has been demonstrated to perpetuate oxidative stress, mitochondrial dysfunction, and cell death. ${ }^{34}$ In the brain, GSH depletion exacerbates oxidative stress, which is a pathogenesis of aging-related neurodegenerative diseases. Cysteine is used to produce GSH. ${ }^{35,36}$ Research indicates that cysteine uptake by excitatory amino acid carrier (EAAC1) is important for neuronal antioxidant function. A previous study showed that mice lacking EAAC1 ${ }^{-/-}$show increased neuronal oxidative stress. ${ }^{37}$ However, this changed when oral $\mathrm{NAC}$ treatment was initiated. In $\mathrm{EAAC}^{-/-}$mice, a model of PD, NAC substantially reduced loss of dopaminergic neurons in the substantia nigra pars compacta, ${ }^{38}$ demonstrating that NAC acts independently from the EAAC1 to cross the neuronal membrane. Other research demonstrated that in patients with major depressive disorder, supplementation with NAC increased the NAC metabolite levels in the anterior cingulate cortex detected by proton magnetic spectroscopy, suggesting that NAC initiates its effects in the brain region. ${ }^{39}$ Thus, NAC administration into the body may help reduce the cytotoxicity caused by ROS generation. Furthermore, NAC may improve endothelial function through its antioxidant properties, which may attenuate Hcy-induced oxidative stress. NAC is well tolerated by most patients, with only mild gastrointestinal symptoms such as flatulence and diarrhea. $^{24,39}$ These studies indicate that NAC can potentially alleviate oxidative stress caused by HHcy. In addition, other pathways are available for detoxifying Hcy further.

\section{Hcy Trans-sulfuration and Remethylation}

Hcy is detoxified in the cells via 2 pathways: transsulfuration and remethylation. With an excess cellular Hcy level, trans-sulfuration converts Hcy into cystathionine with the support of CBS. Cystathionine is then converted into cysteine by cystathionine $\gamma$-lyase. ${ }^{9,40}$ Mutations in genes involved in Hcy metabolism, including CBS, can lead to an increase in tHcy levels. Previous human tissue culture research has demonstrated that Hcy concentration is increased when Hcy metabolism is hindered by mutations in the $C B S$ gene. CBS-deficient $\left(^{-/}\right)$mice have 40 -fold higher total plasma Hcy levels. ${ }^{41-43}$

The remethylation pathway consists of Hcy being remethylated back to Met. Hcy is recycled to Met by 2 enzymes, Met synthase (MS) and betaine-Hcy methyltransferase (BHMT). For both enzymes, the Hcy serves as an acceptor of methyl groups for the maintenance of essential $S$-adenosyl-met-dependent transmethylation reactions. MS 


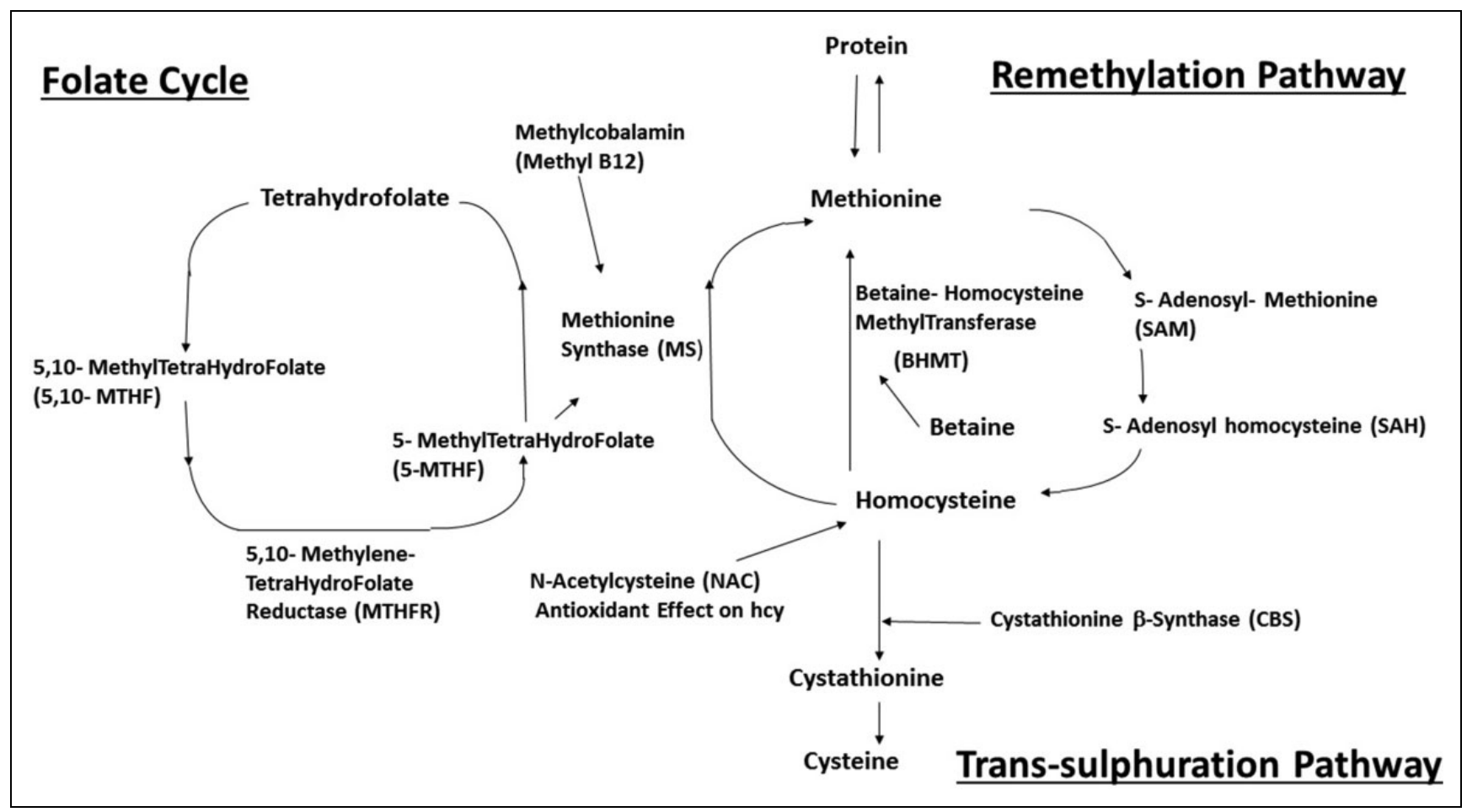

Figure I. The metabolic interactions between 5-methyltetrahydrofolate (5-MTHF), methylcobalamin (methyl-BI2), $\mathrm{N}$-acetylcysteine (NAC), and betaine are shown targeting multiple pathways.

converts 5-methyltetrahydrofolate (5-MTHF), derived from folic acid, into tetrahydrofolate using vitamin B12 as a cofactor. Researchers have developed a genotype mouse model with methyltetrahydrofolate reductase $\left(\mathrm{MTHFR}^{-/-}\right)$ deficiency and demonstrated that these mice have impaired memory. ${ }^{44,45}$

The most common genetic abnormality for Hcy metabolism is a substitution at nucleotide 677 (C677T) of MTHFR. With this genetic mutation, MTHFR activity will decrease by $50 \%$. In the Western countries, $9 \%$ to $17 \%$ of the population was homozygous for this mutation and 30\% to $41 \%$ was heterozygous. ${ }^{11,45,46}$ Homozygosity and heterozygosity for the C677T mutation are the most common MTHFR variants and have been associated with high Hcy. Research has shown that the C677T variant and plasma Hcy levels are significant predictors of reduced regional brain volumes in older adults. With decreased MTHFR, which is the ratelimiting enzyme in the methyl cycle, the level of 5-MTHF production also decreases. 5-MTHF is a predominant form of folate. 5-MTHF together with vitamin B12 is required for the conversion of Hcy to Met by this pathway. ${ }^{10,47}$ Hcy can also obtain a methyl group from betaine, catalyzed by BHMT ${ }^{48,49}$ BHMT-dependent remethylation of Hcy occurs primarily in the liver, kidneys, and lens. The central nervous system lacks BHMT and is therefore dependent on folate and the vitamin B12 pathway for the conversion of Hcy into Met (Figure 1), suggesting enhanced vulnerability of the brain to HHcy. The regulation of BHMT has been shown to affect Hcy concentrations in plasma., 50
The MS and BHMT pathways of Hcy remethylation are considered areas of interest for research when applying the therapeutic cocktail approach to decrease Hcy levels and its toxicity. Folic acid, vitamin B12, or betaine (trimethylglycine) can influence the Met-Hcy cycle and thus enhance the conversion of Hcy into Met. ${ }^{50}$ Betaine has at least 5 different beneficial mechanisms for AD. First, betaine supplementation $(6 \mathrm{~g} / \mathrm{d}$ for $12 \mathrm{wk})$ decreased fasting plasma Hcy by $9 \%$ in obese men and women. ${ }^{51}$ Second, betaine can hinder the short- and long-term memory impairment induced by Hcy. Memory function examinations such as Y-maze and step-down-type passive avoidance test were used as measurements. Third, betaine can inhibit the Hcy-induced matrix matrix metalloproteinase-9 (MMP-9) activity in the frontal cortex, helping to prevent BBB damage. ${ }^{52}$ Fourth, betaine can reduce hyperphosphorylation of tau protein, as hyperphosphorylation of tau protein causes neural fiber entanglement, leading to neurodegeneration. ${ }^{53}$ Fifth, betaine treatment restores expressions of synaptic proteins needed for synaptic plasticity, which are vital to learning and memory. ${ }^{54}$ These factual statements make betaine a promising novel strategy to add in a therapeutic cocktail.

\section{Methylcobalamin}

Insufficient amounts of folate and vitamin B12 limit the conversion of Hcy into Met, influencing the Hcy level. ${ }^{55}$ A vegetarian diet may cause deficiency in vitamin B12, since this vitamin is found only in foods of animal origin. 
Moreover, acquired B12 deficiencies can result from parietal cell and intrinsic factor antibodies (pernicious anemia), malabsorption, age-related gut atrophy, or gastrectomy. ${ }^{56}$ Vitamin B treatment alleviates HHcy by $31.7 \%$ compared with placebo. In effect, this was accompanied by a reduction in the rate of brain atrophy of almost $30 \%$. The prevalence of vitamin B12 deficiency increases with age. Studies have shown that there is a strong association between low B12 levels and risk of dementia. B12 is nontoxic, inexpensive, and well tolerated. ${ }^{4,56,57}$

This study involved a clinical case of an 83-yr-old female with a Mini-Mental State Examination score of 14, demonstrating a moderate degree of cognitive impairment. The study showed that $10,000 \mu \mathrm{g}$ of hydroxocobalamin had no effect on reversing dementia. However, another therapeutic trail of 3,000 $\mu \mathrm{g}$ methylcobalamin and 1,200 $\mu \mathrm{g}$ folic acid daily was given. ${ }^{57}$ Within $2 \mathrm{wk}$, the patient's cognition improved slowly and normal behaviors returned. After 2 mo of methylcobalamin and folic acid, the patient's Mini-Mental State Examination score was back to 29. The patient was able to resume most of her previous activities and social life. ${ }^{57}$ The patient's cognitive impairment improved with methylcobalamin and folic acid. ${ }^{57}$ The cause of relapse during supplementation with hydroxocobalamin is likely an inability to convert hydroxocobalamin into the metabolically active form of methylcobalamin. ${ }^{57}$ Methylcobalamin ultimately enters the MS pathway. Hcy accepts a methyl group from methylcobalamin to be transformed into Met. Methylcobalamin is considered a substance that is biologically active at the cellular level. ${ }^{58}$ This key point makes methylcobalamin a more suitable choice to use in the therapeutic cocktail.

\section{Conclusion}

$\mathrm{AD}$ is the most common cause of dementia in the United States and costs billions of dollars in health care. A substantial amount of research has demonstrated that HHcy leads to cellular oxidative stress and is considered a risk factor for developing AD. HHcy overstimulates NMDA and $\alpha$-amino3-hydroxy-5-methyl-4-isoxazolepropionic acid receptors, resulting in increased levels of cytoplasmic calcium, higher production of free radicals, and activation of caspases, leading to cellular death. The accumulation of these toxic free radicals plays a major role in $\mathrm{BBB}$ disruption through the increased activity of MMPs. However, NAC can restore intracellular GSH levels in the brain, reducing these free radical toxicities. Oral NAC does produce an increase in NAC concentration in the CSF. ${ }^{33}$ NAC also has shown many other benefits in a variety of clinical settings.

Other alternative therapeutic approaches, such as activating the Hcy remethylation pathway, should also be considered for reducing oxidative stress. Both 5-MTHF and methyl B12 are important additives for treating Hhcy, via the remethylation pathway. Clinical evidence has shown that using methylcobalamin can improve a patient's Mini-Mental State Examination, and the patients are able to resume their normal daily activities. In addition, patients who have the MTHFR gene C677T variant will have a decrease in MTHFR enzyme activity, which ultimately decreases 5MTHF production. ${ }^{11,45}$ Therefore, adding 5-MTHF is beneficial. MS depends upon methyl B12 and 5-MTHF in the remethylation pathway. In addition, Hcy is recycled to Met through BHMT. Betaine supplementation has been shown to decrease fasting plasma Hcy, improve memory, decrease MMP activity, and decrease tau hyperphosphorylation. ${ }^{51-53}$

All 5-MTHF, methylB12, NAC, and betaine are available separately as OTC dietary supplements. Therefore, one should be able to make a therapeutic cocktail with 5MTHF, methyl B12, NAC, and betaine for AD treatment. It will be more convenient for patients to have a cocktail treatment rather than taking 4 different supplements. While NAC helps alleviate oxidative stress, 5-MTHF, methyl B12, and betaine can decrease Hcy concentration at the remethylation pathway. This combination of therapeutic cocktail can potentially help $\mathrm{AD}$ pathophysiology by targeting multiple areas simultaneously.

\section{Authors' Contribution}

M.L. reviewed the literature and wrote this article. D.S. edited this article. J.T. and R.S. contributed to the initial idea of the review. All authors read and approved this article.

\section{Declaration of Conflicting Interests}

The author(s) declared no potential conflicts of interest with respect to the research, authorship, and/or publication of this article.

\section{Funding}

The author(s) disclosed receipt of the following financial support for the research, authorship, and/or publication of this article: This work was supported by Ed and Ethel Moore Alzheimer's Disease Research grant 5AZ10 (J.T.) and the Silver Endowment. J.T. holds the Silver Chair in Developmental Neurobiology.

\section{References}

1. Thomas P, Fenech M. Buccal cytome biomarkers and their association with plasma folate, vitamin B12 and homocysteine in Alzheimer's disease. J Nutrigenet Nutrigenomics. 2015; 8(2):57-69.

2. Alzheimer's Association. 2016 Alzheimer's disease facts and figures. Alzheimers Dement. 2016;12(4):459-509.

3. Lao J, Beyer K, Ariza A. The homocysteine pathway: a new target for Alzheimer disease treatment? Drug Dev Res. 2004; 62(3):221-230.

4. Smith AD, Smith SM, de Jager CA, Whitbread P, Johnston C, Agacinski G, Oulhaj A, Bradley KM, Jacoby R, Refsum H. Homocysteine-lowering by B vitamins slows the rate of accelerated brain atrophy in mild cognitive impairment: a randomized controlled trial. PLoS One. 2010;5(9):e12244.

5. Škovierová H, Vidomanová E, Mahmood S, Sopková J, Drgová A, Červeňová T, Halašová E, Lehotský J. The molecular and cellular effect of homocysteine metabolism imbalance on human health. Int J Mol Sci. 2016;17(10):pii: E1733. 
6. Mosharov E, Cranford MR, Banerjee R. The quantitatively important relationship between homocysteine metabolism and glutathione synthesis by the transsulfuration pathway and its regulation by redox changes. Biochemistry. 2000;39(42): 13005-13011.

7. McCaddon A. Homocysteine and cognitive impairment; a case series in a general practice setting. Nutr J. 2006;5:6.

8. Aitken RJ, Flanagan HM, Connaughton H, Whiting S, Hedges A, Baker MA. Involvement of homocysteine, homocysteine thiolactone, and paraoxonase type 1 (PON-1) in the etiology of defective human sperm function. Andrology. 2016;4(2):345-360.

9. Kumar T, Sharma GS, Singh LR. Homocystinuria: therapeutic approach. Clinica Chimica Acta. 2016;458:55-62.

10. Roussotte FF, Hua X, Narr KL, Small GW, Thompson PM; Alzheimer's Disease Neuroimagnig Initiative. The C677T variant in MTHFR modulates associations between brain integrity, mood, and cognitive functioning in old age. Biol Psychiatry Cogn Neurosci Neuroimaging. 2017;2(3):280-288.

11. Sachdev P. Homocysteine and neuropsychiatric disorders. Rev Bras Psiquiatr. 2004;26(1):49-55.

12. Barnham KJ, Masters CL, Bush AI. Neurodegenerative diseases and oxidative stress. Nat Rev Drug Discov. 2004;3(3): 205-214.

13. Ovey IS, Naziroglu M. Homocysteine and cytosolic GSH depletion induce apoptosis and oxidative toxicity through cytosolic calcium overload in the hippocampus of aged mice: involvement of TRPM2 and TRPV1 channels. Neuroscience. 2015;284:225-233.

14. Mattson MP, Shea TB. Folate and homocysteine metabolism in neural plasticity and neurodegenerative disorders. Trends Neurosci. 2003;26(3):137-146.

15. Ho PI, Collins SC, Dhitavat S, Ortiz D, Ashline D, Rogers E, Shea TB. Homocysteine potentiates beta-amyloid neurotoxicity: role of oxidative stress. J Neurochem. 2001;78(2):249-253.

16. Droge W. Free radicals in the physiological control of cell function. Physiol Rev. 2002;82(1):47-95.

17. Esmekaya MA, Tuysuz MZ, Tomruk A, Canseven AG, Yucel E, Aktuna Z, Keskil S, Seyhan N. Effects of cell phone radiation on lipid peroxidation, glutathione and nitric oxide levels in mouse brain during epileptic seizure. J Chemical Neuroanat. 2016;75(Pt B):111-115.

18. Al-Assadi HM, Al-Rajhi SH. Comparative study of human betaine homocysteine methlytransferase (BHMT) between diseases. World J Pharm Pharm Sci. 2016;5(4):491-498.

19. Lipton SA, Kim WK, Choi YB, Kumar S, D'Emilia DM, Rayudu PV, Arnelle DR, Stamler JS. Neurotoxicity associated with dual actions of homocysteine at the N-methyl-D-aspartate receptor. Proc Natl Acad Sci USA. 1997;94(11):5923-5928.

20. Reisberg B, Doody R, Stoffler A, Schmitt F, Ferris S, Mobius HJ. Memantine in moderate-to-severe Alzheimer's disease. N Engl J Med. 2003;348(14):1333-1341.

21. Naziroglu M, Cig B, Ozgul C. Neuroprotection induced by $\mathrm{N}$-acetylcysteine against cytosolic glutathione depletioninduced $\mathrm{Ca} 2+$ influx in dorsal root ganglion neurons of mice: role of TRPV1 channels. Neuroscience. 2013;242:151-60.
22. Kamat PK, Vacek JC, Kalani A, Tyagi N. Homocysteine induced cerebrovascular dysfunction: a link to Alzheimer's disease etiology. Open Neurol J. 2015;9:9-14.

23. Atkuri KR, Mantovani JJ, Herzenberg LA, Herzenberg LA. $\mathrm{N}$-Acetylcysteine - a safe antidote for cysteine/glutathione deficiency. Curr Opin Pharmacol. 2007;7(4):355-359.

24. Wu CC, Zheng CM, Lin YF, Lo L, Liao MT, Lu KC. Role of homocysteine in end-stage renal disease. Clin Biochem. 2012; 45(16-17):1286-1294.

25. Giustarini D, Milzani A, Dalle-Donne I, Tsikas D, Rossi R. $\mathrm{N}$-Acetylcysteine ethyl ester (NACET): a novel lipophilic cell-permeable cysteine derivative with an unusual pharmacokinetic feature and remarkable antioxidant potential. Biochem Pharmacol. 2012;84(11):1522-1533.

26. Robillard JM, Gordon GR, Choi HB, Christie BR, MacVicar BA. Glutathione restores the mechanism of synaptic plasticity in aged mice to that of the adult. PLoS One. 2011;6(5):e20676.

27. Dringen R, Hamprecht B. N-Acetylcysteine, but not methionine or 2-oxothiazolidine-4-carboxylate, serves as cysteine donor for the synthesis of glutathione in cultured neurons derived from embryonal rat brain. Neurosci Lett. 1999; 259(2):79-82.

28. Fishbane S, Durham JH, Marzo K, Rudnick M. N-acetylcysteine in the prevention of radiocontrast-induced nephropathy. J Am Soc Nephrol. 2004;15(2):251-260.

29. Borgstrom L, Kagedal B, Paulsen O. Pharmacokinetics of $\mathrm{N}$-acetylcysteine in man. Eur J Clin Pharmacol. 1986;31(2): 217-222.

30. Rushworth GF, Megson IL. Existing and potential therapeutic uses for $\mathrm{N}$-acetylcysteine: the need for conversion to intracellular glutathione for antioxidant benefits. Pharmacol Ther. 2014;141(2):150-159.

31. Dean O, Giorlando F, Berk M. N-acetylcysteine in psychiatry: current therapeutic evidence and potential mechanisms of action. J Psychiatry Neurosci. 2011;36(2):78-86.

32. Shahripour RB, Harrigan MR, Alexandrov AV. N-acetylcysteine (NAC) in neurological disorders: mechanisms of action and therapeutic opportunities. Brain Behav. 2014;4(2):108-122.

33. Reyes RC, Cittolin-Santos GF, Kim JE, Won SJ, BrennanMinnella AM, Katz M, Glass GA, Swanson RA. Neuronal glutathione content and antioxidant capacity can be normalized in situ by $\mathrm{N}$-acetyl cysteine concentrations attained in human cerebrospinal fluid. Neurotherapeutics. 2016;13(1):217-225.

34. Mischley LK, Conley KE, Shankland EG, Kavanagh TJ, Rosenfeld ME, Duda JE, White CC, Wilbur TK, De La Torre PU, Padowski JM. Central nervous system uptake of intranasal glutathione in Parkinson's disease. NPJ Parkinsons Dis. 2016; 2:16002.

35. Aoyama K, Nakaki T. Glutathione in cellular redox homeostasis: association with the excitatory amino acid carrier 1 (EAAC1). Molecules. 2015;20(5):8742-8758.

36. Sarris J, Oliver G, Camfield DA, Dean OM. Participant characteristics as modifiers of response to N-Acetyl Cysteine (NAC) in Obsessive-Compulsive Disorder. Clin Psychol Sci. 2016;4(6):1104-1111. 
37. Choi BY, Kim IY, Kim JH, Lee BE, Lee SH, Kho AR, Jung HJ, Sohn M, Song HK, Suh SW. Decreased cysteine uptake by EAAC1 gene deletion exacerbates neuronal oxidative stress and neuronal death after traumatic brain injury. Amino Acids. 2016;48(7):1619-1629.

38. Berman AE, Chan WY, Brennan AM, Reyes RC, Adler BL, Suh SW, Kauppinen TM, Edling Y, Swanson RA. N-acetylcysteine prevents loss of dopaminergic neurons in the EAAC1-/mouse. Ann Neurol. 2011;69(3):509-20.

39. Oliver G, Dean O, Camfield D, Blair-West S, Ng C, Berk M, Sarris J. N-acetyl cysteine in the treatment of obsessive compulsive and related disorders: a systematic review. Clin Psychopharmacol Neurosci. 2015;13(1):12-24.

40. Jakubowski H. Quantification of urinary S- and N-homocysteinylated protein and homocysteine-thiolactone in mice. Anal Biochem. 2016;508:118-23.

41. Zang T, Pottenplackel LP, Handy DE, Loscalzo J, Dai S, Deth RC, Zhou ZS, Ma J. Comparison of protein NHomocysteinylation in rat plasma under elevated Homocysteine using a specific chemical labeling method. Molecules. 2016;21(9):1195.

42. Chwatko G, Boers GHJ, Strauss KA, Shih DM, Jakubowski H. Mutations in methylenetetrahydrofolate reductase or cystathionine beta-synthase gene, or a high-methionine diet, increase homocysteine thiolactone levels in humans and mice. FASEB J. 2007;21(8):1707-1713.

43. Kamath AF, Chauhan AK, Kisucka J, Dole VS, Loscalzo J, Handy DE, Wagner DD. Elevated levels of homocysteine compromise blood-brain barrier integrity in mice. Blood. 2006; 107(2):591-593.

44. Jadavji NM, Deng L, Malysheva O, Caudill MA, Rozen R. MTHFR deficiency or reduced intake of folate or choline in pregnant mice results in impaired short-term memory and increased apoptosis in the hippocampus of wild-type offspring. Neuroscience. 2015;300:1-9.

45. Dell'edera D, Tinelli A, Milazzo GN, Malvasi A, Domenico C, Pacella E, Pierluigi C, Giuseppe T, Marcello G, Francesco L, et al. Effect of multivitamins on plasma homocysteine in patients with the 5,10 methylenetetrahydrofolate reductase C677T homozygous state. Mol Med Rep. 2013;8(2):609-612.

46. Roussotte FF, Narr KL, Small GW, Thompson PM. The C677T variant in MTHFR modulates associations between blood- based and cerebrospinal fluid biomarkers of neurodegeneration. Neuroreport. 2016;27(12):948-951.

47. Sobczynska-Malefora A, Cutler J, Rahman Y. Elevated homocysteine with pseudo-homozygosity for MTHFR677T as predisposing factors for transient ischemic attacks: a case report. Metab Brain Dis. 2016;31(5):1205-1208.

48. Selhub J, Troen A, Rosenberg IH. B vitamins and the aging brain. Nutr Rev. 2010;68(Suppl 2):S112-S118.

49. Williams KT, Schalinske KL. Homocysteine metabolism and its relation to health and disease. Biofactors. 2010;36(1):19-24.

50. Rajdl D, Racek J, Trefil L, Stehlik P, Dobra J, Babuska V. Effect of folic acid, betaine, vitamin B6, and vitamin B12 on homocysteine and dimethylglycine levels in middle-aged men drinking white wine. Nutrients. 2016;8(1):34.

51. Steenge GR, Verhoef $P$, Katan MB. Betaine supplementation lowers plasma homocysteine in healthy men and women. J Nutr. 2003;133(5):1291-1295.

52. Kunisawa K, Nakashima N, Nagao M, Nomura T, Kinoshita S, Hiramatsu M. Betaine prevents homocysteine-induced memory impairment via matrix metalloproteinase- 9 in the frontal cortex. Behav Brain Res. 2015;292:36-43.

53. Sun J, Wen S, Zhou J, Ding S. Association between malnutrition and hyperhomocysteine in Alzheimer's disease patients and diet intervention of betaine. J Clin Lab Anal. 2016;1-7.

54. Chunjie N, Huijuan N, Zhao Y, Jianzhao W, Xiaojian Z. Betaine reverses the memory impairments in a chronic cerebral hypoperfusion rat model. Neurosci Lett. 2016;615:9-14.

55. de Koning EJ, van der Zwaluw NL, van Wijngaarden JP, Sohl E, Brouwer-Brolsma EM, van Marwijk HW, Enneman AW, Swart KM, van Dijk SC, Ham AC, et al. Effects of two-year vitamin B12 and folic acid supplementation on depressive symptoms and quality of life in older adults with elevated Homocysteine concentrations: additional results from the B-PROOF study, an RCT. Nutrients. 2016;8(11):pii: E748.

56. Lachmann RH, Briddon A. Homocysteine and methylmalonate: when should I measure them and what do they mean? Pract Neurol. 2016;16(4):328-333.

57. Rietsema WJ. Unexpected recovery of moderate cognitive impairment on treatment with oral methylcobalamin. J Am Geriatr Soc. 2014;62(8):1611-1612.

58. Ahmed MA. Metformin and vitamin B12 deficiency: where do we stand? J Pharm Pharm Sci. 2016;19(3):382-398. 